\title{
Possibility of comb-type rf shield structure for high-current accelerators
}

\author{
Y. Suetsugu, M. Shirai, and K. Shibata \\ High Energy Accelerator Research Organization (KEK), 1-1 Oho, Tsukuba, Ibaraki, 305-0801, Japan
}

(Received 9 August 2003; published 15 October 2003)

\begin{abstract}
A novel rf shield structure for bellows chambers or gate valves in high-current accelerators is proposed. The rf shield has no thin fingers, but nested comb teeth instead at the inner surface of the beam duct. The comb-type rf shield has a higher thermal strength structurally than the conventional finger-type one. A simulation also indicates its lower impedance. Especially, the leak of a TE modelike higher order mode through the axial slits, which causes mainly heating of these components, is almost suppressed due to thick teeth. An abrasion test in a vacuum using a test piece of the comb-type rf shield shows less dust generation compared to the finger type. Another preliminary test transmitting $508 \mathrm{MHz}$ microwaves through a prototype $\mathrm{rf}$ shield in the atmosphere found no abnormal heating or arcing. A trial model of bellows chambers with the comb-type rf shield will be installed and tested using a real beam in the KEK $B$-factory positron ring this year.
\end{abstract}

DOI: 10.1103/PhysRevSTAB.6.103201

PACS numbers: 29.20.-c, 29.90.+r

\section{INTRODUCTION}

In an accelerator vacuum system, several vacuum components, like bellows chambers and gate valves, which would have a large axial gap otherwise, are usually equipped with rf (radio frequency) shield structures inside. The rf shield bridges the gap to reduce the beam impedance and to avoid the heating of components due to the extra HOM (higher order modes) excited there. The conventional $\mathrm{rf}$ shield is a finger type, which consists of many narrow and thin fingers surrounding the inner surface of the beam duct [1-7]. As a typical example, the inside view of a finger-type rf shield for the KEKB (KEK $B$ factory) is shown in Fig. $1[6,7]$. Each finger has a width and thickness of 5.5 and $0.2 \mathrm{~mm}$, respectively. The gap between adjacent fingers is $0.5 \mathrm{~mm}$. The finger-type $\mathrm{rf}$ shield has been available in many various accelerators so far without any conspicuous problems.

Recent high-power accelerators, that is, highluminosity colliders with beam currents higher than $1 \mathrm{~A}$ (or a bunch current larger than about $1 \mathrm{~mA}$ ) and short bunch lengths of less than about $10 \mathrm{~mm}$, such as the KEKB and the PEP-II [8,9], however, put much more severe conditions than ever before on the rf shield structure. The storage current of ampere orders causes a high wall current density. The high bunch current and the short bunch length lead to intense HOM excitation at various vacuum components. These result in heating of the fingers due to Joule loss. Especially, the TE modelike HOM, which has axial components of magnetic fields, easily couples with the space between the rf shield and the outside structure through the axial slits between the fingers. This also heats up the fingers as well as the outside structure. Actually, heating of the bellows or gate valves has been observed at KEKB and PEP-II [8-10]. In the worst case, the rf shield was damaged by excess heating, as shown in Fig. 2, near strong HOM sources. The broken and bent fingers, as shown in the figure, sometimes blocked the injection beam and deteriorated the accelerator performance. For future higher luminosity accelerators with storage currents of over $10 \mathrm{~A}[11,12]$, the heating or damage of the finger-type rf shield can be a serious hardware problem.

Considering these circumferences, we propose here a new-type rf shield, that is, a comb-type rf shield, for highcurrent accelerators. The rf shield is no more than the finger type, and will have a higher thermal strength than the conventional one. From the viewpoint of beam impedance, the calculated loss factor was lower than that of the conventional finger type. A simulation also indicated that leakage of the TE modelike HOM to the outside is drastically reduced compared to the thin finger type. Preliminary tests using a trial model, that is, an abrasion test in a vacuum and a heating test transmitting $508 \mathrm{MHz}$ microwaves in the atmosphere, were performed and gave promising results. In the following, first the structure of the comb-type rf shield is described, and then the

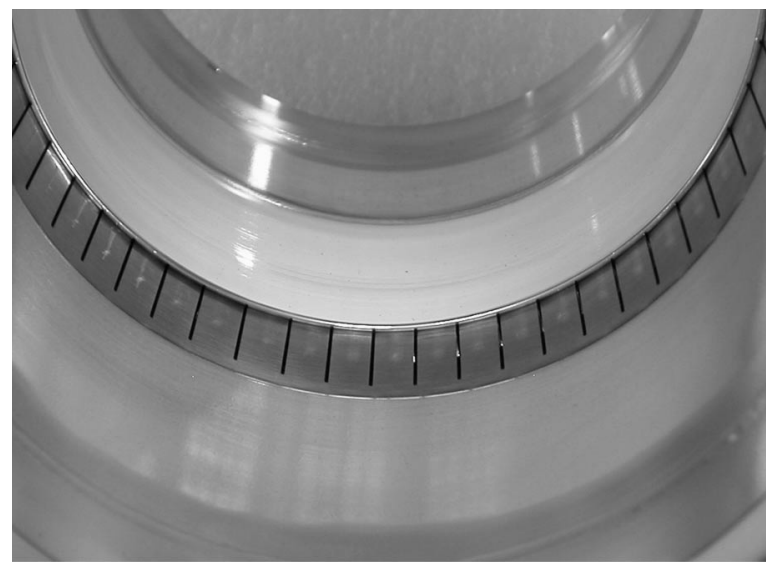

FIG. 1. Inside view of the finger-type $\mathrm{rf}$ shield (bellows for KEKB). 


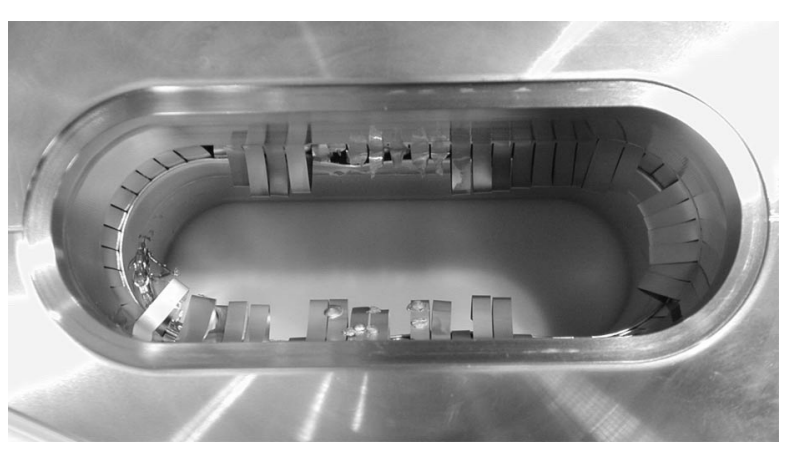

FIG. 2. Example of a damaged finger-type rf shield observed at KEKB.

calculated rf properties and results concerning the preliminary tests are presented and discussed.

\section{STRUCTURE}

An inside view of a comb-type rf shield for a circular beam duct is presented in Fig. 3(a), corresponding to the finger type in Fig. 1. The rf shield is no more than thin
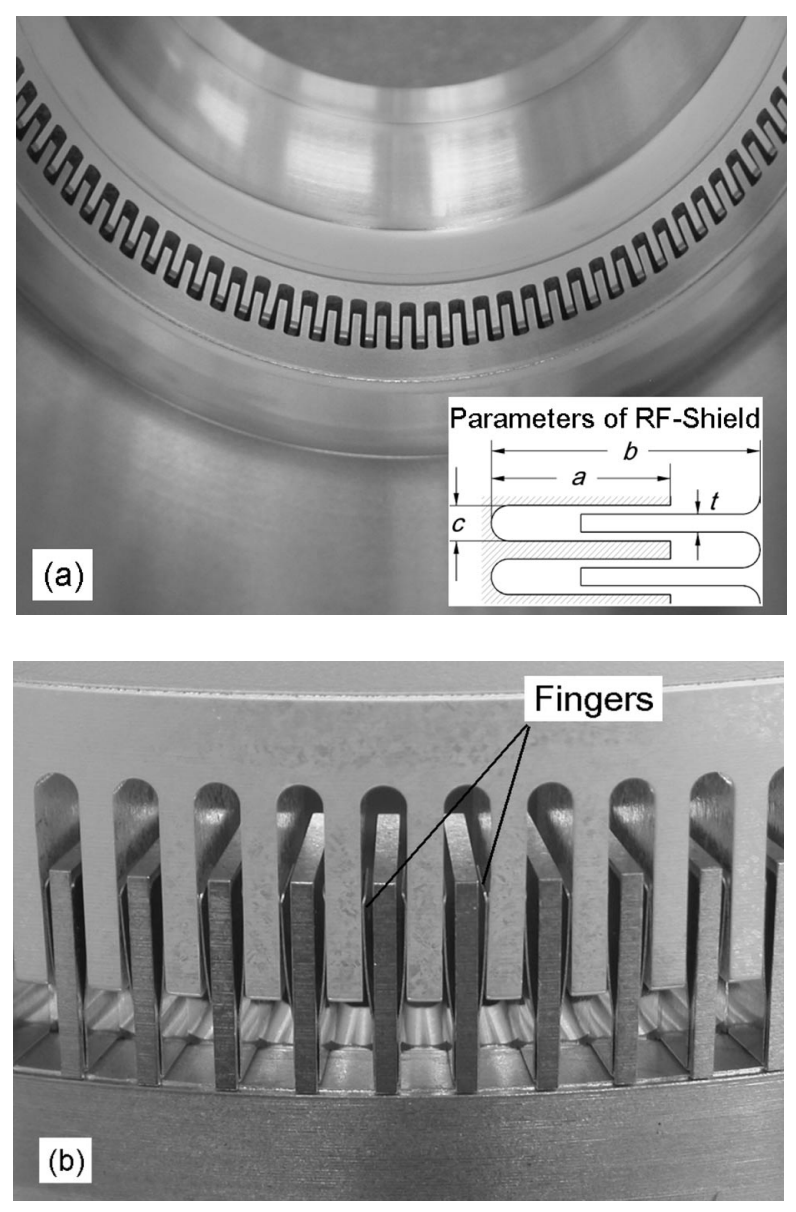

FIG. 3. (a) Inside view of a comb-type rf shield, i.e., a nested comb. (b) Outside of the comb-type rf shield. The small fingers are between the nested teeth. fingers, but nested comb teeth. In a trial model, the length [(a) in the sketch in Fig. 3(a), the same way in the following], the width $(t)$, and the radial thickness of a comb tooth are 10,1 , and $10 \mathrm{~mm}$, respectively. The gap between adjacent teeth $(c)$ is $2 \mathrm{~mm}$ and, therefore, the gap between the nested teeth is $0.5 \mathrm{~mm}$. At the nominal position, the total length of the $\mathrm{rf}$ shield structure $(b)$ is $15 \mathrm{~mm}$. For the circular beam duct with an inner diameter of $94 \mathrm{~mm}$, for example, the number of teeth is 100 . The combs are made of pure copper and are easily machined.

In principle, the high-frequency wall current accompanied with a bunched beam flows via capacitance between nested teeth (a gap of $0.5 \mathrm{~mm}$ ). To ensure the flow of $\mathrm{dc}$ or a low-frequency wall current, small fingers (backup fingers) are prepared at the outer half between the nested teeth, as shown in Fig. 3(b). The backup fingers have an important role to block the leakage of HOM (see Sec. IIIC). The backup fingers are made of berylliumcopper alloy or silver-coated Inconel alloy with a thickness of $0.1 \mathrm{~mm}$ and a width of $5 \mathrm{~mm}$. The current flowing in this finger is expected to be small compared to the present finger-type rf shield (Fig. 1), since the finger is not at the inner surface, but $5 \mathrm{~mm}$ behind the surface. The contact force, therefore, can be less than that of the conventional finger type (1 N per a finger typically) and is set to about $0.6 \mathrm{~N}$ per a finger in the trial model.

The advantages of the new rf shield are as follows:

(i) The rf shield has a high thermal strength. Since the width of a tooth $(t)$ is thick $(1 \mathrm{~mm})$ and can be made of copper, its thermal conductivity is larger by a factor of 40 than that of the conventional finger $(\mathrm{Be}-\mathrm{Cu}$ finger with a width, thickness, and length of 5.5, 0.2, and $20 \mathrm{~mm}$, respectively [6,7]). The heat load to the rf shield will be easily transferred to a cooling channel. Even if damaged, for example, by discharging or accidental irradiation of synchrotron radiation, the results will not be such a disaster as in the case of using the finger type (see Fig. 2).

(ii) There is no radial step on the inner surface of the beam duct. That leads to a low impedance, as described later. The conventional finger-type rf shield should have a radial step (or bump), in principle, to make sliding contact points of fingers [see Fig. 1], and sometimes the fingers should have a curvature to enforce the contact force.

(iii) The TE modelike HOM hardly goes out through the $\mathrm{rf}$ shield due to the large thickness $(10 \mathrm{~mm})$ of the teeth, as described later.

(iv) There is no sliding point on the inner surface of the beam duct, which otherwise could be a source of arcing or heating in the case of a bad electric contact.

(v) The rf shield can fit for various cross sections of beam duct, such as a racetrack, an oval, a polygon, or an antechamber (key hole).

On the other hand, the axial stroke of the rf shield is limited structurally. For the trial model described above, the available strokes should be $+3 \mathrm{~mm}$ (expansion) and 
$-4 \mathrm{~mm}$ (contraction), which are smaller than that of the conventional finger type, typically $\pm 10 \mathrm{~mm}$. Bending at the rf shield of $20 \mathrm{mrad}$ will be possible, like the usual finger-type one, although tilting is hardly expected to occur intrinsically. The limitations of the stroke or bending, however, can be tolerable if the alignment and the manufacturing accuracy of beam ducts can be well supervised. The temperature rise of the beam duct, of course, should be controlled. This structural restriction will not raise any problem in an application to the gate valve, where the stroke is originally small and no bending or tilting is necessary, in principle.

\section{RF PROPERTIES}

\section{A. Loss factors}

Using the MAFIA simulation code, the loss factors of the comb-type rf shield were calculated and compared to the finger-type one for several conditions. For the comb type, the simulation model had a total length of $200 \mathrm{~mm}$, as presented in Fig. 4. The mesh size was 0.25 and $0.5 \mathrm{~mm}$ for the transverse and axial directions, respectively. The outer half part of the nested teeth was short-circuited assuming backup fingers [Fig. 3(b)]. The loss factors were calculated by the indirect method. For the finger-type rf shield, on the other hand, the model had a length of $2000 \mathrm{~mm}$. The transverse and axial mesh sizes were 0.5 and $0.8 \mathrm{~mm}$, respectively. The loss factors were calculated by the direct method. The gap between the fingers is not included (it is too detailed), but only a radial bump with a height and an axial length of 1 and $20 \mathrm{~mm}$, respectively, is considered [see Fig. 1]. For both cases, the model has a circular cross section with a diameter of $94 \mathrm{~mm}$, assuming application to the present KEKB positron ring [10,13].

The dependence of the loss factors $\left[k\left(\mathrm{~V} \mathrm{C}^{-1}\right)\right]$ for the comb-type and finger-type rf shields as a function of the bunch length $\left[\sigma_{z}(\mathrm{~mm})\right]$ are shown in Fig. 5. The loss factor of the comb type is smaller than that of the finger type by a factor of 3 or 4 . The ratio of the loss factor of the comb type to that of the finger type, furthermore, de-

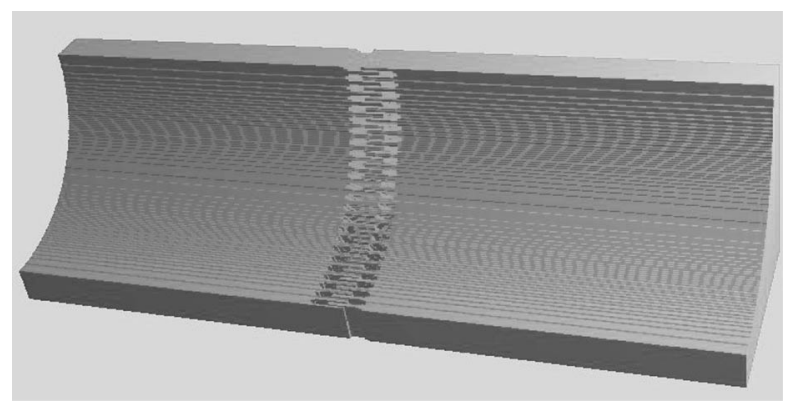

FIG. 4. Simulation model of the comb-type rf shield to estimate the loss factors. The total length is $200 \mathrm{~mm}$ and the mesh size is 0.25 and $0.5 \mathrm{~mm}$ for the transverse and axial directions, respectively.

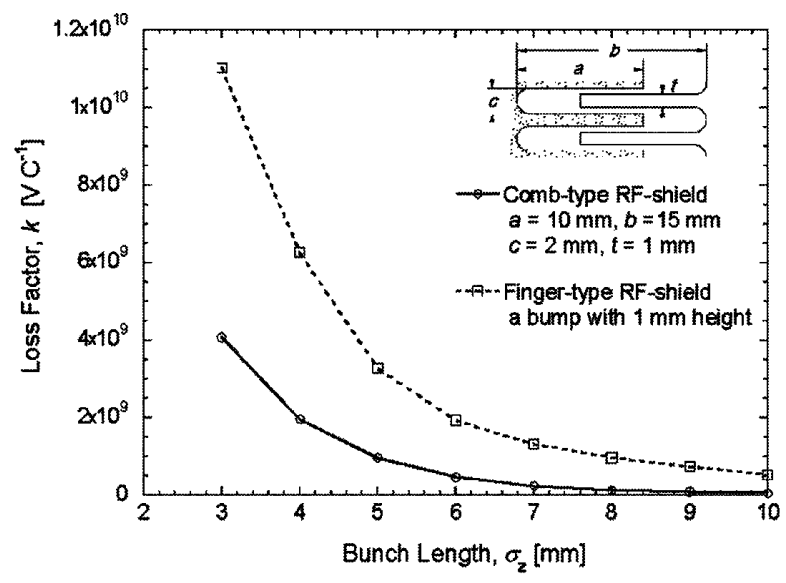

FIG. 5. Dependence of loss factors $(k)$ of the comb-type and the finger-type rf shields on the bunch length $\left(\sigma_{z}\right)$ for a circular beam duct with a diameter of $94 \mathrm{~mm}$. For the comb type, the tooth has a length ( $a$ in the sketch, the same hereafter) of $10 \mathrm{~mm}$, a width $(t)$ of $1 \mathrm{~mm}$, and a thickness of $10 \mathrm{~mm}$. The gap between the teeth $(c)$ is $2 \mathrm{~mm}$ and the length of the rf shield (b) is $15 \mathrm{~mm}$. For the finger type, only a radial bump with a height and an axial length of 1 and $20 \mathrm{~mm}$, respectively, is considered.

creased with increasing the bunch length, which means that the comb-type rf shield is very available, even for the low-frequency modes, although the comb-type rf shield is first intended for the high-frequency HOM caused by short bunches.

The longitudinal wake functions $\left[w_{\|}\left(\mathrm{V} \mathrm{C}^{-1}\right)\right]$ for both rf shields are presented in Fig. 6. The wake has the shape of an inductive impedance source. The amplitude for the comb type is smaller than that of the finger type. However, the wake function of the comb-type rf shield seems to include a particular mode with a frequency of about $14 \mathrm{GHz}$. Actually, a mode with that frequency is excited between the nested fingers, as shown later.

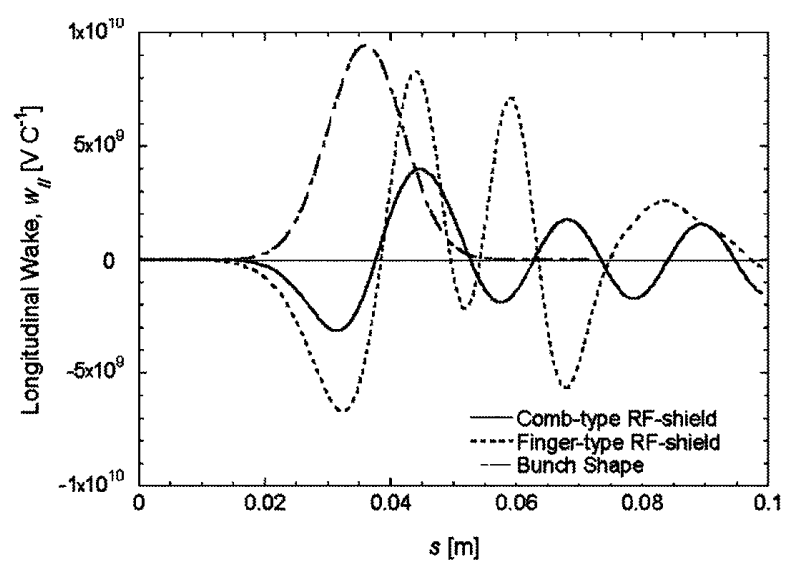

FIG. 6. Longitudinal wake function $\left(w_{\|}\right)$of the comb-type and the finger-type rf shields for the same condition as in Fig. 5. 


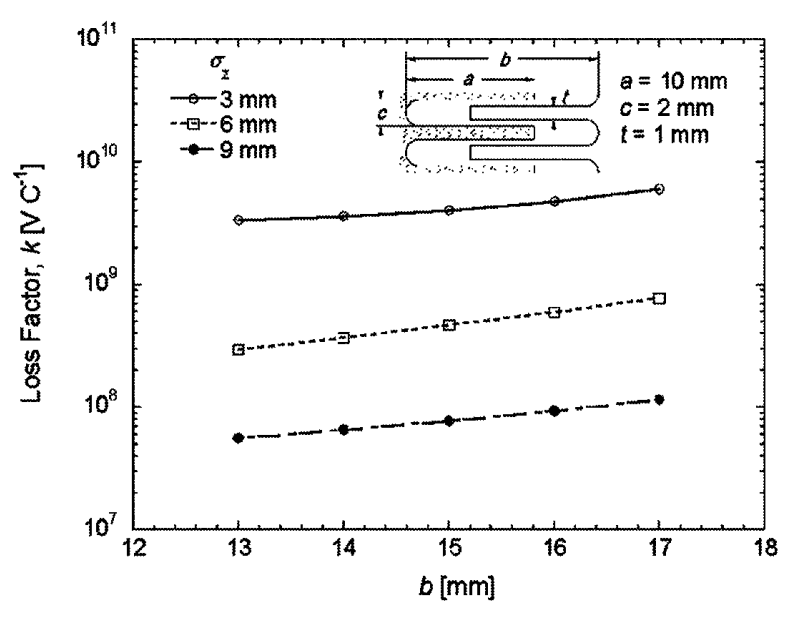

FIG. 7. Dependence of the loss factors $(k)$ on the length of the rf shield $(b)$ for three bunch lengths $\left(\sigma_{z}\right)$.

The loss factors during expansion $(+2 \mathrm{~mm})$ and contraction $(-2 \mathrm{~mm})$ were calculated, and the results are presented in Fig. 7 as a function of the length of the $\mathrm{rf}$ shield $[b(\mathrm{~mm})]$ for three bunch lengths $\left(\sigma_{z}\right)$. The loss factor is reduced upon contracting the rf shield. This is because the length of the nested part $(2 a-b)$ increases; that is, the capacitance becomes large, and the slot length $(b-a)$ decreases at the same time. Another simulation also found that a longer tooth length $(a)$ has smaller loss factors for the same length of the rf shield $(b)$. The loss factors as a function of gaps between adjacent teeth $(c)$ are shown in Fig. 8 for three bunch lengths $\left(\sigma_{z}\right)$. Increasing the gap increases the loss factor, because the flow of wall current is more steered. A narrower gap should be better from the viewpoint of beam impedance, but machining of the teeth would be difficult and the mechanical strength of the tooth would weaken. A long tooth has

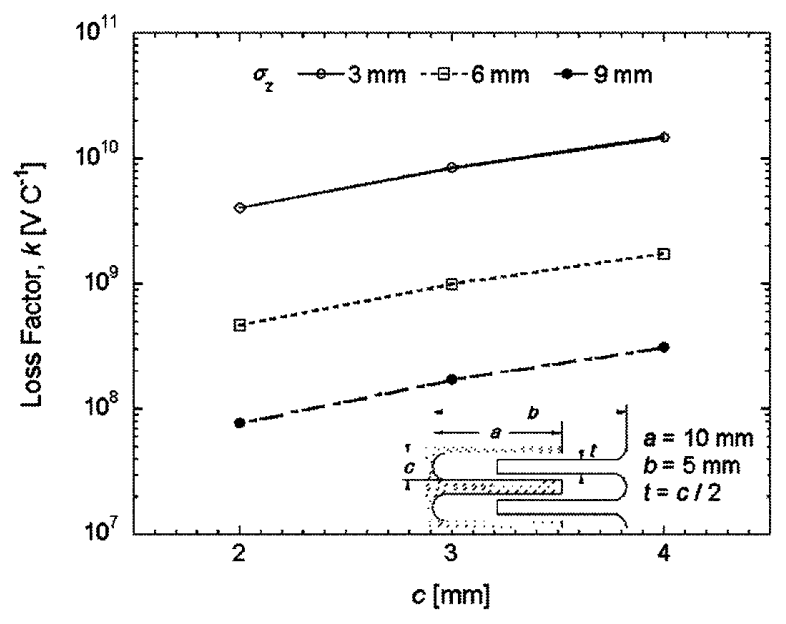

FIG. 8. Dependence of the loss factors $(k)$ on the width of the gap between the teeth $(c)$ for three bunch lengths $\left(\sigma_{z}\right)$. the same problem and, furthermore, makes the bending tolerance small.

\section{B. Electric field between teeth}

Figure 9 presents the change of the electric field intensities in the gaps between the adjacent teeth and the nested teeth ( $g_{1}$ and $g_{2}$ in the sketch) as a function of the depth $[d(\mathrm{~mm})]$. The electric fields in the figure are the peak values just after a bunch with a length of $3 \mathrm{~mm}$ and a charge of $1 \mathrm{C}$ has passed the center of a duct with a diameter of $94 \mathrm{~mm}$.

In gap $g_{1}$, the intensity of the electric field decreases rapidly as the position goes outside. Only the electric field of the $x$ component is plotted in the figure, but the other components ( $y$ and $z$ ) also behave similarly. Since gap $g_{1}$ is the only way for the HOM to go outside, because the backup fingers block it in gap $g_{2}$ [see Fig. 3(b)], it indicates that the HOM will hardly leak outside through the thick teeth, which is also demonstrated in the following section.

In gap $g_{2}$, on the other hand, the maximum electric field does not change very much with the depth. The temporal behavior of the electric field in gap $g_{2}$ at a depth (d) of $3 \mathrm{~mm}$ is shown in Fig. 10. The mode has a frequency of about $14 \mathrm{GHz}$, and seems to be an eigenmode, excited in the gap. The mode, however, damps rapidly with time. The intensity decreased to one-half within $0.3 \mathrm{~ns}$, as indicated in the figure. For accelerating cavities driven by $508 \mathrm{MHz}$ microwaves, for example, the rf bucket spacing is at least $2 \mathrm{~ns}$. The mode, therefore, will sufficiently damp until the succeeding bunch passes the $\mathrm{rf}$ shield. Rapid damping means that the frequency is higher than the cutoff frequency of the mode for the present duct.

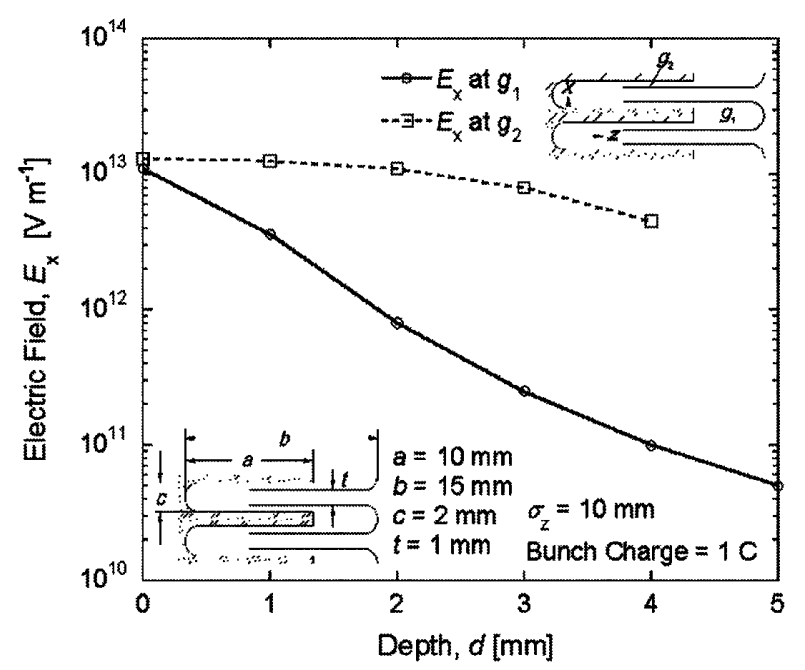

FIG. 9. Electric field intensities along the depth $(d)$ of the gap between the adjacent teeth $\left(g_{1}\right)$ and between the nested teeth $\left(g_{2}\right)$. 


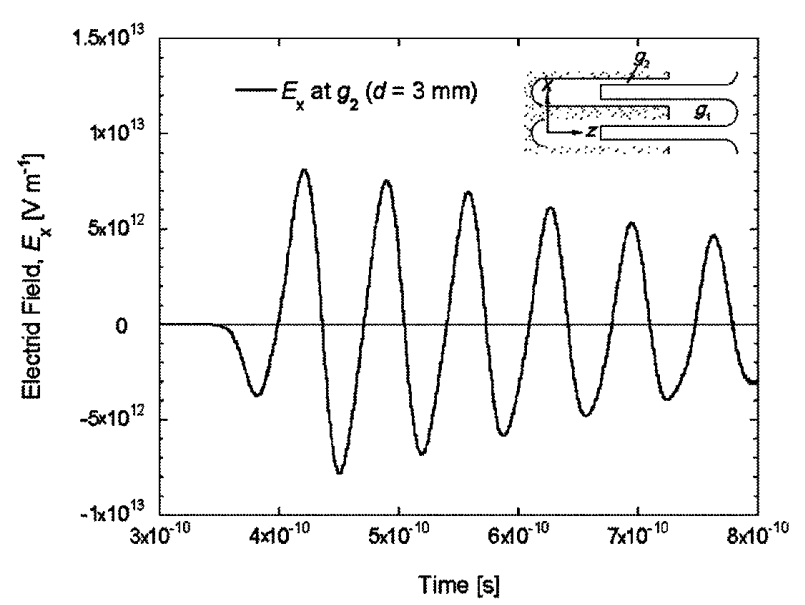

FIG. 10. Temporal behavior of the electric field $\left(E_{x}\right)$ in the gap $g_{2}$ at a depth $(d)$ of $3 \mathrm{~mm}$.

With realistic beam parameters, those of the Super KEKB for example, the bunch charge is about $2 \times$ $10^{-8} \mathrm{C}$ [12]. In that case the maximum electric field is about $2 \times 10^{5} \mathrm{~V} \mathrm{~m}^{-1}$ in gap $g_{2}$ as expected from Fig. 9 . Since the gap width is $0.5 \mathrm{~mm}$, the gap voltage is $100 \mathrm{~V}$. The voltage is well below the Kilpatrick condition [14] and discharging (arcing) does not occur in the gap. On the other hand, the product of the distance $(0.5 \mathrm{~mm})$ and the frequency $(14 \mathrm{GHz})$ is $7 \mathrm{GHz} \mathrm{mm}$, and the multipactoring also does not occur for even a gap voltage of $100 \mathrm{~V}$ [15].

\section{HOM outside of the rf shield}

The HOM intensities outside of the rf shield were calculated for both types of rf shields. The calculation models were the same as the previous one used to estimate the loss factor of the comb-type rf shield. The cross sections of the models and the monitoring point are shown in Fig. 11. The solid and the broken lines are the cross sections for the comb-type and the finger-type rf shields, respectively. The sizes of the comb type were the same as those of the trial model described above $(a=$ $10 \mathrm{~mm}, b=15 \mathrm{~mm}, c=2 \mathrm{~mm}$, and $t=1 \mathrm{~mm}$ ). In the calculation, the outer half part of the nested teeth was short-circuited assuming a backup finger. For the finger type, on the other hand, the width, thickness, and length of the finger were $8,0.5$, and $20 \mathrm{~mm}$, respectively. The gap between the fingers was $1 \mathrm{~mm}$. These sizes were somewhat different from the actual one due to the limitation of the used mesh size. The monitoring point is indicated by " $\mathrm{X}$ " in the figure. A beam with a length $\left(\sigma_{z}\right)$ of $4 \mathrm{~mm}$ passed at the origin along a line normal to the plane. A small block was placed on the inner wall just upstream of the rf shield as a source of HOM. The calculated axial $(z)$ components of the magnetic fields $\left[B_{z}(\mathrm{~T})\right]$ are presented in Fig. 12 for the cases of the comb types (with and without backup fingers) and the finger type. The intensities are those from about 0.1 to $0.5 \mathrm{~ns}$ just after the bunch passing.

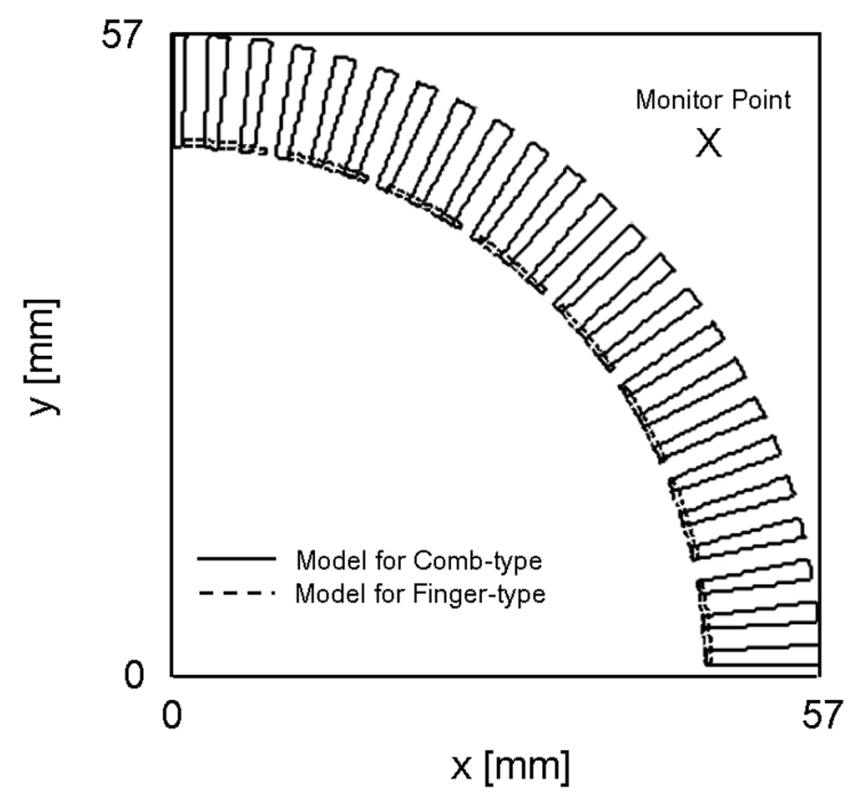

FIG. 11. Cross sections of the models to calculate the HOM intensities outside of the rf shield. The whole model is the same as in Fig. 4. The solid and the broken lines are those of the comb-type and the finger-type rf shields, respectively. The monitoring position is indicated by "X."

As shown in the figure, the intensities for the comb type with backup fingers were much smaller than those for the finger type by 1 order of magnitude. The horizontal $(x)$ and vertical $(y)$ components of electric fields behaved similarly. Since these components represent the TE modelike HOM, it can be said that the TE modelike HOM is well damped by a thick comb-type rf shield.

Note here that the intensity of $B_{z}$ for the case of a comb-type rf shield without backup fingers is almost the same as that of the finger type. That means that the leakage of the TE modelike HOM is comparable to the case of the finger type if the comb type has no backup

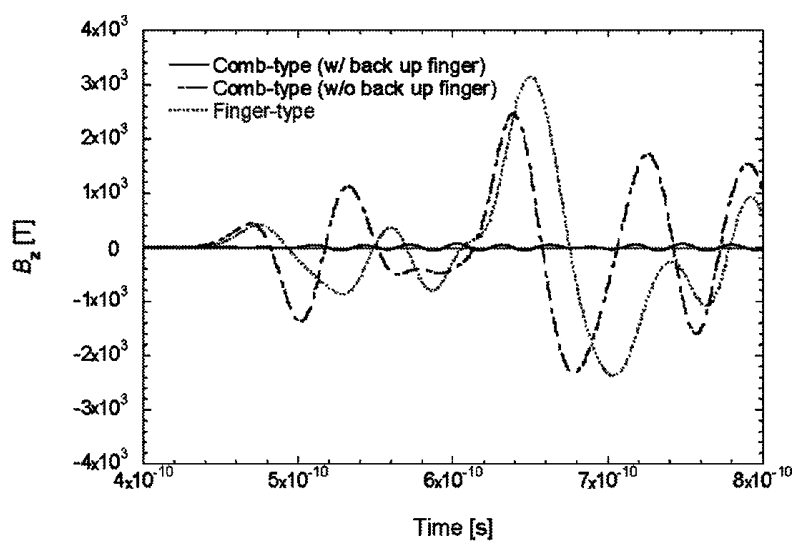

FIG. 12. Intensities of the axial ( $z$ ) components of the magnetic fields for the comb-types (with and without backup fingers) and the finger-type rf shields. 


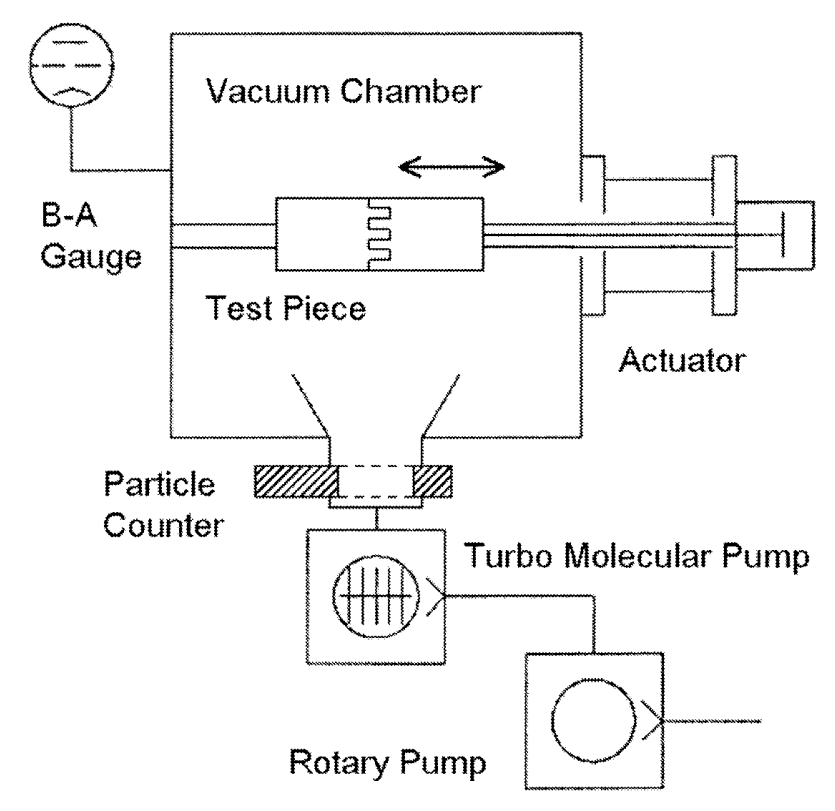

FIG. 13. Setup diagram for an abrasion test in a vacuum.

fingers. The mode between the nested teeth (see Fig. 10) seems to go through the gap and be emitted to the outside. It was found that the backup fingers, that is, short cutting the nested teeth, are important to sufficiently suppress the HOM outside of the rf shield. The backup fingers, or some other measures to short-circuit the nested teeth just outside (see Fig. 17 later), will be indispensable for the combtype $\mathrm{rf}$ shield in high-current machines. The present structure of backup fingers, however, is somewhat complex. Considering the application to gate valves, furthermore, the sliding part (i.e., dust source) should be avoided as much as possible. It is a key issue in the future to find a way to short-circuit the nested teeth surly with a simple structure and less abrasion.

\section{PRELIMINARY TESTS}

\section{A. Abrasion test in a vacuum}

A schematic diagram of the abrasion test in a vacuum is presented in Fig. 13 [16]. A test piece of the rf shield was installed in a vacuum chamber which had been evacuated down to less than $1 \times 10^{-3} \mathrm{~Pa}$. The test piece has five teeth and, therefore, ten sliding fingers. The tooth was silver-coated pure copper, and had the same size as the trial model $(a=10 \mathrm{~mm}, c=2 \mathrm{~mm}$, and $t=1 \mathrm{~mm})$. The finger was a silver-coated Inconel alloy with a width and thickness of 5 and $0.1 \mathrm{~mm}$, respectively. The contact force was about $0.6 \mathrm{~N}$ per finger. The fingers performed reciprocating motion with a stroke of $5 \mathrm{~mm}$ with a time interval of about $5 \mathrm{~s}$. The particle counter used was a M-25S (Ushio Co. Ltd., Japan), which counts particles by a laser scattering method in a vacuum. The wavelength of the semiconductor laser was $780 \mathrm{~nm}$. The size of a particle could be classified into five ranges between 0.27 and

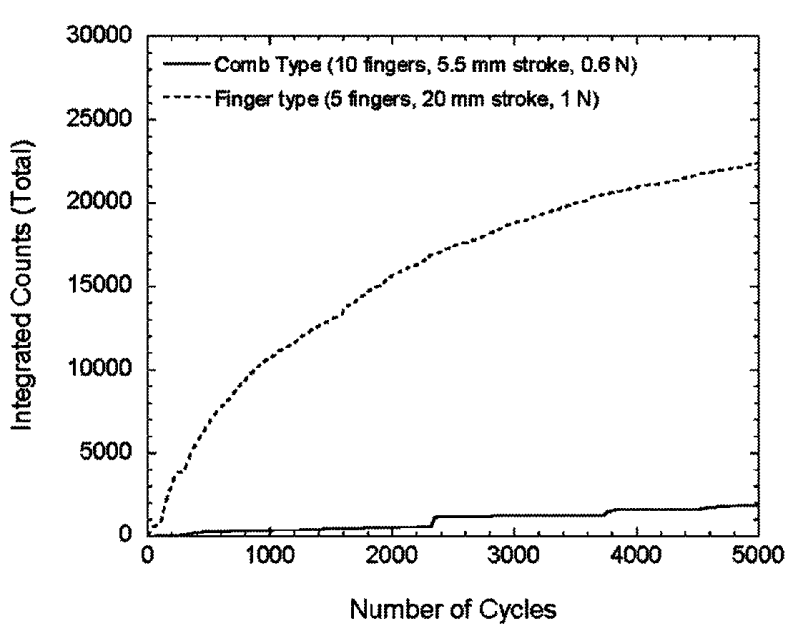

FIG. 14. Integrated total amounts of particles $(>0.27 \mu \mathrm{m})$ for the comb-type and the finger-type rf shields.

$2.0 \mathrm{~mm}$. The particles dropped from the test piece were gathered by a cone and passed the detector of the particle counter.

Figure 14 shows the result of the integrated amounts of the measured total particles $(>0.27 \mu \mathrm{m}$ ) for the new $\mathrm{rf}$ shield (solid line) as a function of the number of cycles. The generation of particles was large at the initial stage, and then decreased. That must have been due to the oxide layer on the surface formed in the atmosphere. Most of the particles were in the range of 0.27 and $0.5 \mathrm{~mm}$. The small steps in the figure are bursts of particles. As a reference, the result for the finger-type rf shield [16] is also plotted in the figure by a broken line. The stroke, the number of fingers, and the contact force were $20 \mathrm{~mm}, 5$, and about $1 \mathrm{~N}$, respectively. The fingers (Be-Cu alloy) were sandwiched between a plate (silver-coated stainless steel) and spring fingers (silver-coated Inconel alloy). The structure and the material were the same as those used for the bellows chambers of the present KEKB [6,7]. As shown in the figure, the comb-type rf shield generates fewer particles by a factor 100 than does the finger type. Major reasons would be that the contact force of the fingers is weak, and that the fingers are also coated by silver. Since dust trappinglike phenomena have been hardly observed, even in the electron ring of KEKB, actually once a day at most, dust generation from the comb-type rf shield should not cause any trouble.

\section{B. Heating test}

The heating of the comb-type rf shield by the wall current was examined by transmitting high-power microwaves [6]. The TEM mode of the coaxial transmission line was suitable for the test because the wall current has mainly an axial component, in principle, just like a real wall current. The necessary microwave powers to induce rms wall current densities equivalent to the several 
TABLE I. Several examples of the operation parameters [bunch length, $\sigma_{z}(\mathrm{~mm})$; bunch spacing, $\tau_{b}(\mathrm{~ns})$; and total current, $\left.I_{b}(\mathrm{~A})\right]$ and necessary input power $\left(P_{\text {in }}\right)$ to obtain the equivalent rms wall current densities using a coaxial line transmitting $508 \mathrm{MHz}$ microwaves.

\begin{tabular}{ccccc}
\hline \hline $\begin{array}{c}\text { Bunch length } \\
\sigma_{z}(\mathrm{~mm})\end{array}$ & $\begin{array}{c}\text { Bunch spacing } \\
\tau_{b}(\mathrm{~ns})\end{array}$ & $\begin{array}{c}\text { Total current } \\
I_{b}(\mathrm{~A})\end{array}$ & $\begin{array}{c}\text { Input power } \\
P_{\text {in }}(\mathrm{kW})\end{array}$ & Notes \\
\hline 4 & 2 & 2.6 & 15 & Designed KEKB \\
6 & 8 & 2.6 & 40 & Present KEKB \\
3 & 2 & 9.4 & 260 & Design of Super KEKB \\
\hline \hline
\end{tabular}

operation conditions are presented in Table I. For a Gaussian bunch, the necessary input power $\left[P_{\text {in }}(\mathrm{W})\right]$ to give the same rms wall current density can be written as follows [6]:

$$
P_{\text {in }}=\frac{Z_{i} c_{0} \tau_{b}}{4 \pi^{3 / 2} \sigma_{z}} I_{b}^{2} \ln \frac{r_{2}}{r_{1}}
$$

where $\tau_{b}(\mathrm{~s})$ and $I_{b}(\mathrm{~A})$ are the bunch spacing and the beam current, respectively. The $Z_{i}=377 \Omega$ is the impedance of the vacuum and $c_{0}=3 \times 10^{8} \mathrm{~m} \mathrm{~s}^{-1}$ is the velocity of light. The $r_{1}(=20 \mathrm{~mm})$ and $r_{2}(=47 \mathrm{~mm})$ are the inner and outer diameters of the coaxial line, respectively. The rms wall current densities are the most deeply related parameters to heating.

Figure 15 shows a partial layout of the experimental setup, including the trial model of the $\mathrm{rf}$ shield. Utilized is a $508 \mathrm{MHz}$ klystron used for the rf accelerating cavities in a KEKB, which can provide a maximum output power of $1 \mathrm{MW}$. The $\mathrm{TE}_{10}$ mode in the rectangular waveguide (WR-1500 type) was transformed to the TEM mode in the coaxial line (WX-152D type) by a coaxial coupler. The input power and the reflected power were monitored by power meters through calibrated directional couplers. A $50 \Omega$ coaxial transmission line was formed by a trial model $(\phi 94 \mathrm{~mm})$ and an inner $\operatorname{rod}(\phi 40 \mathrm{~mm}) . \mathrm{cw}$ microwaves were transmitted through the model and finally absorbed in a dummy load with a capacity of $80 \mathrm{~kW}$. The structure of the comb-type rf shield was the same as that described above. The temperatures at

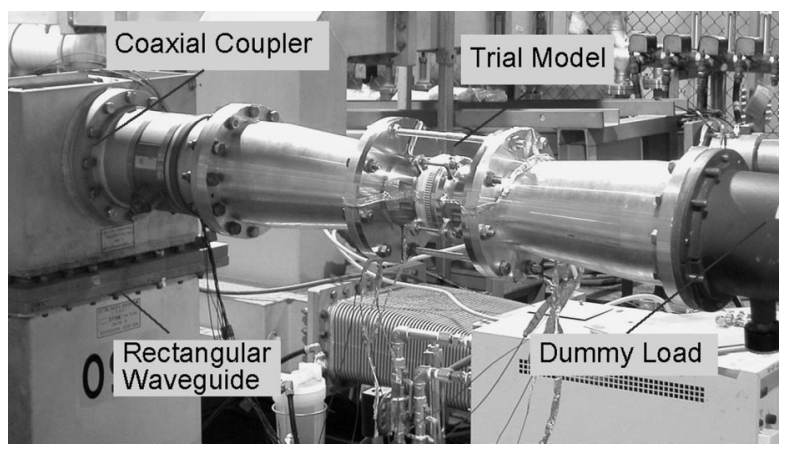

FIG. 15. Setup of the heating test transmitting $508 \mathrm{MHz}$ microwaves. eight points near to the root of the teeth and two points on the body of the duct were measured by thermocouples. The rf shield was insulated thermally by aluminum sheets to clearly observe the temperature rise.

In the experiment, the input power was stepped up by $10 \mathrm{~kW}$ from 20 to $80 \mathrm{~kW}$ in cw, the limit of the dummy load. Modulation on the microwave output wave form, however, made it possible to test with a flattop power of up to $400 \mathrm{~kW}$. The modulation was a series of trapezoid pulses with a rise time of $2 \mathrm{~s}$, a flattop time of $1 \mathrm{~s}$, and a falling time of $2 \mathrm{~s}$. The pulse was generated every $10 \mathrm{~s}$ and the average power was about $1 / 4$ of the flattop power. The flattop power was increased by $100 \mathrm{~kW}$ steps up to $400 \mathrm{~kW}$. At $400 \mathrm{~kW}$, for example, the rms current density was larger by 1.5 times compared to those of the designed beam current in the Super KEKB (see Table I). The experiments were performed while changing the length of the rf shield $(b)$ from 12 to $18 \mathrm{~mm}$.

The temperature rises at several power levels are presented in Figs. 16(a) and 16(b) for the case $b=15 \mathrm{~mm}$. The power was maintained for about $15 \mathrm{~min}$ at each power level, and the temperature rises between 5 and $10 \mathrm{~min}$ were recorded every $1 \mathrm{~min}$. Two data were those at the upstream and downstream sides of the opposed comb teeth. The measured temperatures at ten points changed similarly, but those in the figure are the average of the temperature at the root of the teeth (eight points). The dashed line in the figure is the calculated temperature rise, considering Joule loss of about $100 \mathrm{~mm}$ centered on the $\mathrm{rf}$ shield, where the lengths of copper and stainless steal were 22 and $78 \mathrm{~mm}$, respectively. In the calculation, the rf shield was assumed to be completely thermally insulated. Although the data were scattered due to a small temperature rise, the measured temperature rise was almost in agreement with the expected ones. The main heat source was at the stainless-steel part of the model chamber. Actually, in an experiment using a second trial model made of copper and aluminum, the temperature rise decreased to less than one-fourth. Almost the same temperature rises were observed for other lengths of the $\mathrm{rf}$ shield $(b)$.

The reflected power was always less than about $2 \%$ of the input power, which was comparable to that from the dummy load, itself. This means that the rf shield structure had a negligible impedance for $508 \mathrm{MHz}$ microwaves. 

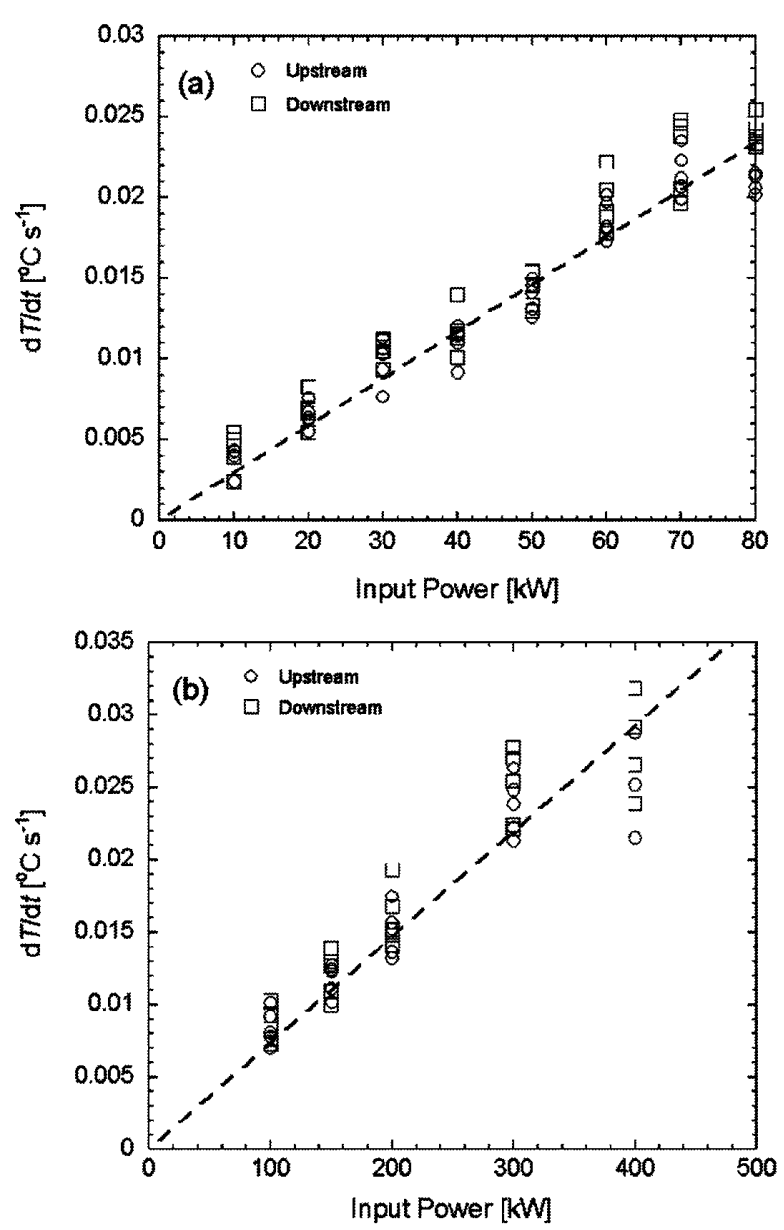

FIG. 16. Temperature rises of the rf shield part as a function of the input power of (a) 20-80 kW and (b) 100-400 kW, where the dashed line is those calculated from the Joule loss at $100 \mathrm{~mm}$ region centered on the rf shield.

After the experiments, the inside and outside of the $\mathrm{rf}$ shield were checked, and no trace of abnormal local heating or arcing was found. However, the arcing should be given attention concerning several points. First, the experiment was not performed in a vacuum, but in the atmosphere. Arcing likely could not occur compared to the case of a vacuum. Second, the electric field on the inner surface was much smaller than that generated by a real bunched beam. It should also be noted that the frequency of the HOM should be higher than that used in the test. Although the simulated electric field intensities were lower than the criterion of arcing, as mentioned before, the final results will wait for tests in real accelerator rings.

\section{FUTURE PLAN}

A simulation and a preliminary test of the comb-type $\mathrm{rf}$ shield gave promising results, as described above. The availability to accelerators, however, should be studied using a real beam, where the frequency spectrum of the

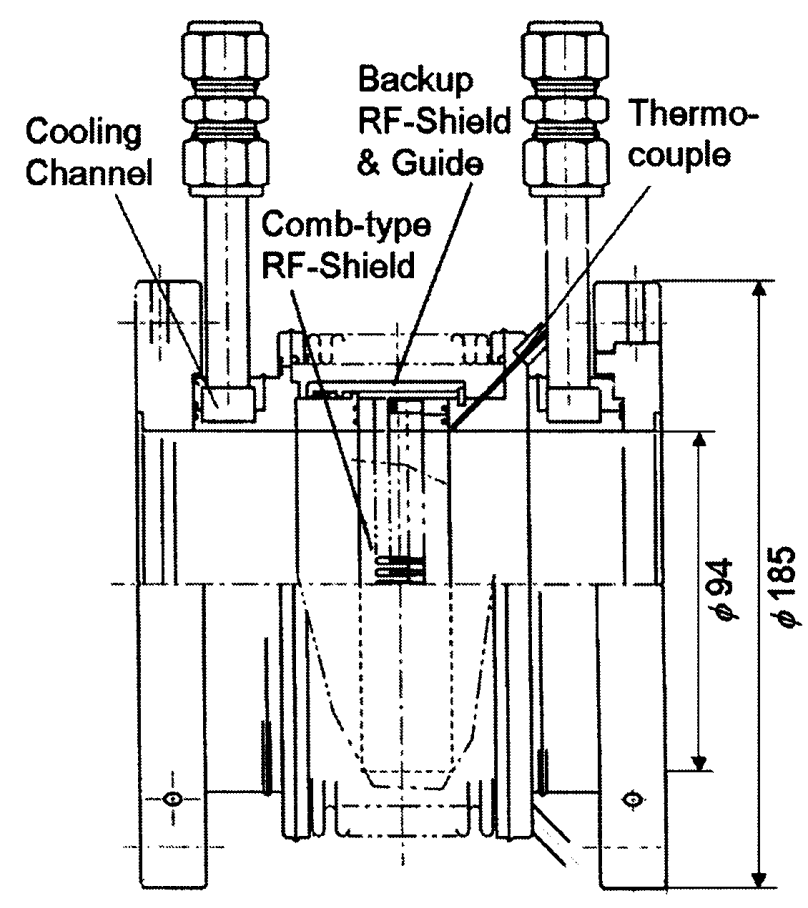

FIG. 17. Prototype of the bellows chamber with the combtype rf shield designed for the KEKB positron ring.

excited HOM and the wall current are different from the preliminary test. Two prototypes of bellow chambers with this new rf shield are presently manufactured and will be installed in the KEKB positron ring this year to see the basic properties of the comb-type rf shield. A schematic of the structure of the prototype is presented in Fig. 17. The length and the diameter are 160 and $185 \mathrm{~mm}$, respectively, and will be replaced by the conventional bellows. The rf shield has a circular cross section $(\phi 94 \mathrm{~mm})$. In the prototype, an additional $\mathrm{rf}$ shield (a backup rf shield), which also has a role to guide the movement, is prepared just outside of the main combtype rf shield for safety. Simplifying the double rf shield structure will be a subject in the next step. The bellows chamber is equipped with two cooling channels. The temperature of the rf shield will be measured by two thermocouples just near to the teeth, as shown in the figure. The arcing will be detected by a pressure gauge installed near the bellows. Furthermore, a discussion on application to the gate valves has just started. Developing a reliable rf shield should be a key point to realize future high-luminosity machines, such as the Super KEKB. Deliberate research and development will be continued in the future.

\section{ACKNOWLEDGMENTS}

The authors would like to thank the members of the KEKB accelerator group for their useful discussions. The authors especially express their deep appreciation to T. Kageyama, K. Ebihara, and H. Nakanishi of the 
KEKB rf group for their valuable suggestions and kind instructions during the rf experiments.

[1] R. Ballion, J. Boster, W. Giesske, H. Hartwig, D. Jagnow, R. Kose, J. Kouptsidis, G. Schumann, and M. Schwartz, Vacuum 41, 1887 (1990).

[2] LEP Vacuum Group, Vacuum 41, 1882 (1990).

[3] W. Unterlerchner, Vacuum 41, 1920 (1990).

[4] J.T. Seeman, in Proceedings of the PAC'95, Dallas (IEEE, Piscataway, NJ, 1995), p. 486.

[5] C. Belser, J. D. Berg, H. Kerns, M. McDaniel, M. Mugge, W. Stoeffl, A. Kulikov, N. Kurita, E. Reuter, and U. Wienands, in Proceedings of the EPAC'96, Sitges (IOP, Bristol, 1996), p. 1090.

[6] Y. Suetsugu, K. Ohshima, and K. Kanazawa, Rev. Sci. Instrum. 67, 2796 (1996).

[7] Y. Suetsugu, K. Kanazawa, and K. Ohshima, Vacuum 47, 629 (1996).

[8] K. Akai et al., Nucl. Instrum. Methods Phys. Res., Sect. A 499, 191 (2003).
[9] J. Seeman et al., in Proceedings of the EPAC'02, Paris, http://epaper.kek.jp/e02/default.html, p. 434 (to be published).

[10] K. Kanazawa, S. Kato, Y. Suetsugu, H. Hisamatsu, M. Shimamoto, and M. Shirai, Nucl. Instrum. Methods Phys. Res., Sect. A 499, 66 (2003).

[11] J.T. Seeman, in Proceedings of the PAC'01, Chicago (IEEE, Piscataway, NJ, 2001), p. 305.

[12] Y. Ohnishi, in Proceedings of the KEK, 2003 (KEK, Tsukuba, 2003), p. 1.

[13] K. Kanazawa, S. Kato, Y. Suetsugu, H. Hisamatsu, M. Shimamoto, M. Sato, and M. Shirai, Appl. Surf. Sci. 169-170, 715 (2001).

[14] W. D. Kilpatrick, Rev. Sci. Instrum. 28, 824 (1957).

[15] A. J. Hatch and H. B. Williams, Phys. Rev. 112, 681 (1958).

[16] Y. Suetsugu, K. Kanazawa, M. Kawahara, Y. Harada, and M. Kaneko, in Proceedings of the 11th Symposium on Accelerator Science and Technology, Harima (Japan), 1997 (Spring-8, Hyogo, 1997), p. 362. 